\title{
Influence of Minority Carrier Transport in the Optical Properties of Double Barrier Diodes
}

\author{
I. Camps ${ }^{\dagger}$, A. Vercik ${ }^{\ddagger}$, L. F. dos Santos ${ }^{\dagger}$, and Y. Galvão Gobato \\ ${ }^{\dagger}$ Departamento de Física, Universidade Federal de São Carlos, São Carlos-SP, Brazil and \\ ${ }^{\ddagger}$ FZEA, Departamento de Ciências Básicas, Universidade de São Paulo, Pirassununga-SP, Brazil
}

Received on 4 April, 2005

\begin{abstract}
The aim of this work is to study the importance of minority carrier transport in double barrier diodes (DBD). We propose a theoretical model capable to describe the photoluminescence properties observed in $G a A s-A l_{0.35} G a_{0.65} A s$ double barrier diodes with the increase of temperature. In this model, we considered that the minority carries (holes) photocreated at the contact of the structure diffuse, drift and then tunnel to the well. To study the influence of previous transport mechanisms, we solved the continuity equation under the influence of an applied bias and an excitation laser light. The theoretical photocurrent and photoluminescence calculations agree quite well with the experimental observations. Within the approaches of our model, we are able to simulate the minority holes mobility from the photocurrent measurements suggesting the use of the experimental technique as a probe of the carrier mobility.
\end{abstract}

Keywords: Minority carrier transport; Double barrier diodes; $G a A s-A l_{0.35} G a_{0.65} A s$

\section{INTRODUCTION}

Photoluminescence (PL) is a powerful technique that has been employed to characterize bulk materials [1] and as a probe of transport processes, which take place in double barrier structures. This technique has been used to study the charge build-up in the quantum wells [2], transport and accumulation of optically excited minority carriers in the same device [3] and more recently as an imaging spectroscopy to map out the center-of-mass wave functions of an exciton confined in a GaAs quantum dot [4]. The PL signal, which provide the most important information on the mechanisms of operation of resonant tunneling diodes, arise from recombination between electrons, which tunnel into the quantum well (QW), and holes photocreated in the $n^{+} G a A s$ region of the structure and then tunneled into the QW. Therefore, the transport processes in the structure plays an important role in the photoluminescence of resonant tunneling structures.

In the present paper, we have studied transport and optical properties of GaAs/AlGaAs double barrier diodes. As it is well-known, the PL intensity should decrease as temperature increases because the nonradiative mechanisms become dominant [5]. We have observed an unexpected enhancement of the measured PL intensity with increasing of temperature for biased diodes. In order to simulate the PL emission spectra, many methods had been developed using different approaches. In this work, we developed a model that take into account two different contributions to the hole population in the well to simulate the integral PL intensity as a function of temperature and the applied bias in order to reproduce the anomalous behavior of the PL spectra obtained experimentally. This model agree quite well with the experimental measurements.

The rest of this paper is organized as follows. In section II, we present the theoretical model developed to describe the observed PL anomalous behavior. In sections III and IV we present the results and the conclusions respectively.

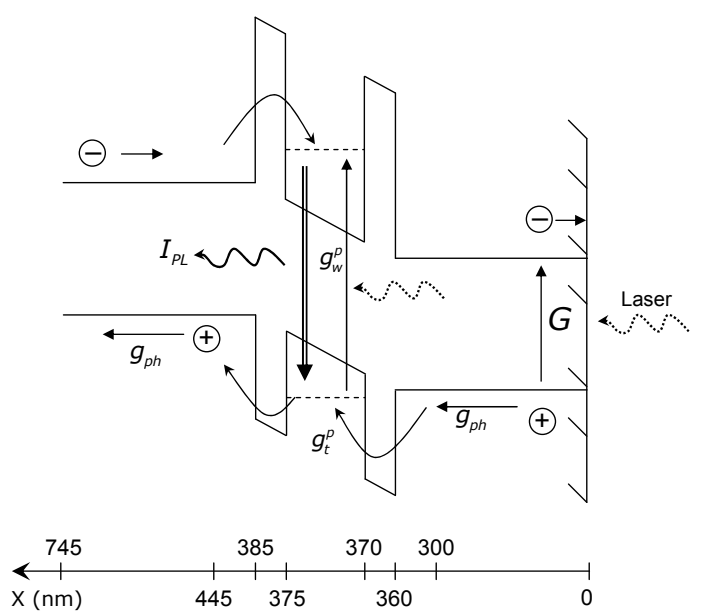

Fig. 1 Schematic diagram of conduction and valence band of the heterostructure (not to scale) and processes involved in the PL emission $\left(I_{P L}\right)$. The curly-dashed arrow represents the incident laser light. The electron-hole pairs are generated at the contact with a rate $G$ and directly in the well with rate $g_{w}^{p}$. The photocreated holes drift and diffuse $\left(g_{p h}\right)$ toward the barrier and tunnel into the well. The doubleline arrow represents the PL emission due to the recombination of both type of holes.

\section{THEORETICAL MODEL}

As it is well known [6], the PL intensity is proportional to the total generation rate of holes in the quantum well, $g^{p}$, and to the quantum efficiency, $\eta$; thus, $I_{P L} \sim \eta g^{p}$. There are two different contributions to the total hole generation rate in the well: holes directly generated in the well, $g_{w}^{p}$, and holes photogenerated in the contact that tunnel inside the well $g_{t}^{p}$.

In order to develop a model that describe appropriately the phenomena involved in the photoluminescence processes, we considered that, in steady state, all the holes that reach the barrier surface tunnel inside the well. 
A schematic representation of these processes and the conduction and valence bands of the heterostructure are shown in Fig. 1.

To obtain an appropriate expression for the tunneling holes, we first calculate the photogenerated holes $\left(g_{p h}\right)$ in the contact. To do that, the continuity equation in one dimension for the excess of carriers at the collector within the drift-diffusion approximation (expression (1)) must be solved in the steady state regime with the appropriate boundary conditions (expression (1)).

$$
\begin{gathered}
0=\frac{\partial \Delta p}{\partial t}=G-\frac{\Delta p}{\tau_{p}}-\mu_{p} E \frac{\partial \Delta p}{\partial x}+D_{p} \frac{\partial^{2} \Delta p}{\partial x^{2}}, \\
\Delta p=\left\{\begin{array}{cl}
G \tau_{p} & x=0 \\
0 & x=\infty
\end{array}\right.
\end{gathered}
$$

In expression (1) the parameters are: the particle lifetime $\tau_{p}$, the hole mobility $\mu_{p}$, the hole diffusion coefficient $D_{p}$, the applied electric field $E$ and the electron-hole pair generation rate $G$.

A solution for this kind of problem can be written as [7]:

$$
g_{p h}=G e^{-\lambda x}
$$

where $G$ is the generated electron-hole rate calculated using the Beer's law [1]

$$
G=(1-R) F(x) e^{-\alpha x},
$$

$F$ is the incident photon flux, $R$ is the reflectivity coefficient, $\alpha$ is the absorption coefficient which temperature dependence was took as been proportional to $\left(h v-E_{g}\right)^{1 / 2}$ with $h v$ been the laser excitation energy, $E_{g}$ the $G a A s$ band-gap [8] and $\lambda$ is a characteristic length that depends on the hole mobility $\left(\mu_{p}\right)$, the diffusion length $\left(L_{p}\right)$ and the applied electric field $E$

$$
\lambda=\frac{1}{2 L_{p}}\left[\sqrt{\left(\frac{E}{E_{c}}\right)^{2}+4}-\frac{E}{E_{c}}\right]
$$

with

$$
L_{p}=\sqrt{\tau_{p} \mu_{p} k_{B} T} \quad \text { and } \quad E_{c}=\frac{L_{p}}{\mu_{p} \tau_{p}} .
$$

The electric field $E_{c}$ represents the critical field for which the minority holes will drift under an applied bias a distance equal to the distance it diffuses due to concentration gradients. At this critical field, both drift and diffusion are equivalent mechanism. There are two transport limiting cases: only diffusion $\left(E \ll E_{c}\right)$ and only drift $\left(E \gg E_{c}\right)$. For each case the characteristic length is different

$$
\lambda=\left\{\begin{array}{cc}
\frac{1}{L_{p}} & E \ll E_{c} \\
\frac{e E}{k_{B} T} & E \gg E_{c} .
\end{array} .\right.
$$

It is worth noting that as the doped layer is five time greater than the spacer layer, we considered only one type of mobility for both. The hole mobility temperature dependence in doped $\mathrm{GaAs}$ was measured for temperatures above $80 \mathrm{~K}$ given an approximately $T^{-1}$ dependence [9]. Nevertheless, mobility would be expected to decrease as temperature goes to zero. Thus, the following semi-empirical temperature dependence for the hole mobility was assumed

$$
\mu_{p}(T)=\left(\frac{a}{T^{2}}+b T\right)^{-1}
$$

where $a$ and $b$ are fitting parameters in our model.

Assuming that all the photogenerated carries tunnel inside the well

$$
g_{t}^{p}=g_{p h}
$$

The holes directly generated in the well can be calculated using the expression

$$
g_{w}^{p}=G e^{-\alpha x} \quad \text { with } x=360 n m
$$

The PL integrated intensity can then be calculated as

$$
I_{P L}=\eta\left(g_{w}^{p}+g_{t}^{p}\right)
$$

Here, the temperature dependence of the quantum efficiency $\eta$ was took as $\sim p_{1} T^{-p_{2}}$ with $p_{1}$ and $p_{2}(>0)$ been fitting parameters.

In order to simulate the experimental results, the expressions (2) and (10) were used to describe the temperature and applied bias of the photocurrent and PL integrated intensity respectively.

\section{RESULTS}

The samples used in this work are symmetric $n-i-n$ $G a A s / A l_{0.35} G a_{0.65} A s$ double-barrier diodes grown by molecular beam epitaxy. The heterostructures consist in two $i-A l_{0.35} G a_{0.65} A s$ barriers (10nm thick) and an $i-G a A s$ well ( $5 \mathrm{~nm}$ width). The double-barrier system is enclosed by a $60 \mathrm{~nm} i-G a A s$ and a $300 \mathrm{~nm} n^{+}-G a A s\left(10^{18} \mathrm{~cm}^{-3}\right)$ layers grown on both sides of the structure. Annular contacts and an optical window of $1.420 \times 10^{-3} \mathrm{~cm}^{2}$ in the top of $500 \mu m \times 600 \mu m$ mesas allow photocurrent and optical measurements under applied voltages. The samples were mounted in a close cycle cryostat. The spectra were recorded by a Spex $500 M$ single spectrometer. A coherent $\mathrm{Ar}^{+}$ion laser was used as excitation source to create minority holes and the PL signal was detected by a photocounting system connected to a thermoelectrically cooled $R 5108$ Hamamatsu photomultiplier. 


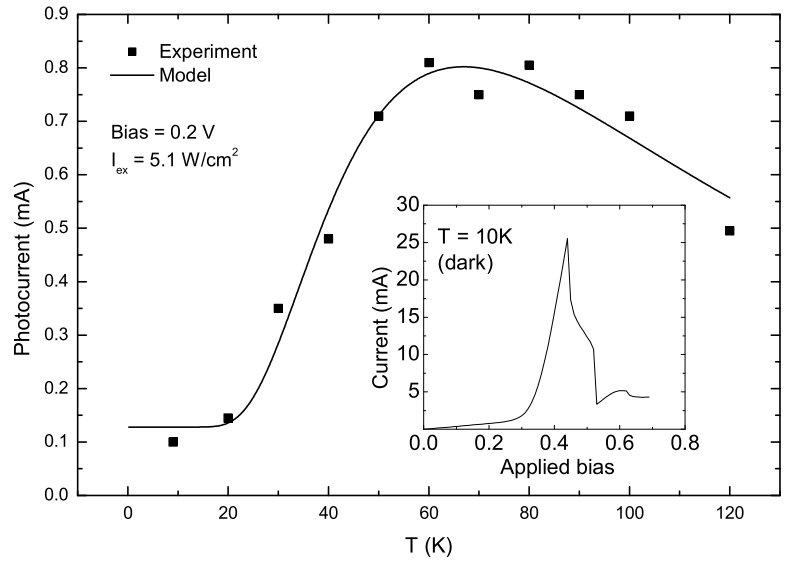

Fig. 2. Measured (points) and calculated (solid line) photocurrent for an applied bias of $0.2 \mathrm{~V}$ and $5.1 \mathrm{~W} / \mathrm{cm}^{2}$ excitation intensity. In the inset, a dark current-voltage characteristic at $10 \mathrm{~K}$.

The $I(V)$ characteristic of the structure in the dark is presented in the inset of figure 2 . The electron resonance peak is observed at $0.47 \mathrm{~V}$. We have measured the photocurrent under low laser intensity (to avoid deformations in the $I(V)$ characteristic) as function of the temperature. In figure 2, we show the experimentally obtained photocurrent and the calculated using expression (2) at $0.2 \mathrm{~V}$ near of the onset of electron resonance. As in our model we are only considering the influence of minority carrier transport, it is necessary an applied voltage low enough to avoid the tunneling of electrons. When a higher voltage is applied, the electrons begin to tunnel and the holes photocurrent is screened and the decreasing expected temperature dependence behavior is obtained. The points in the figure 2 were calculated from the difference between the measured current under the laser light $\left(j_{i}\right)$ and the measured current in the dark $\left(j_{d}\right)$. Experimentally, we have observed an increasing of photocurrent with temperature which is in good agreement with our theoretical model.

In the calculation of $g_{p h}$, we used the expression (7) to simulate the temperature dependence of the mobility in the $n^{+}-$GaAs layer. In figure 3 we show the simulated minority hole mobility. In order to get an estimate of the mobility values obtained, the experimental measured data from Ref. [9] are also shown in the same graph. As can be seen from it, the method used to obtain the temperature dependence of the minority hole mobility from the measured photocurrent gives satisfactory results. This shows the possibility to use this technique in order to determine indirectly the minority holes mobility. Nevertheless, it is worth noting again that the applied bias has to be low enough to avoid the influence of tunneling electrons on the photocurrent. Only photocurrent measurements done at low bias are available for minority holes mobility determination.

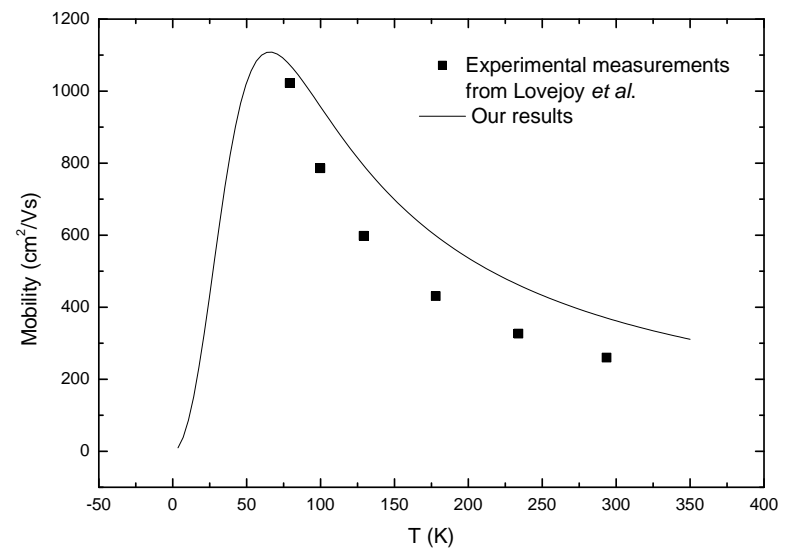

Fig. 3. The solid line represents the theoretical simulated minority hole mobility. The dots are experimentally measured data from Lovejoy et al. (Ref. [9]).

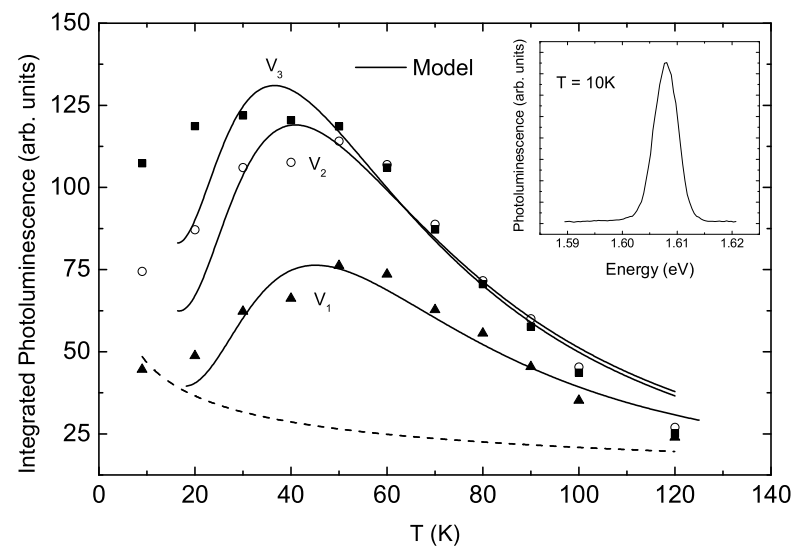

Fig. 4. Experimental and calculated integral photoluminescence intensity as a function of temperature for different applied bias $V_{1}=$ $200 \mathrm{mV}, V_{2}=250 \mathrm{mV}$ and $V_{3}=300 \mathrm{mV}$, respectively, and $5.1 \mathrm{~W} / \mathrm{cm}^{2}$ excitation intensity. In the inset, a typical QW PL spectrum.

A typical quantum well photoluminescence spectrum for the structure at $10 \mathrm{~K}$, excited at $2.7 \mathrm{eV}$, is shown in the inset of figure 4. The PL spectra are obtained with the structure biased with the same voltages of photocurrent measurements. It arises from recombination between electrons in the lower confined level of conduction band well and holes in the lowest heavy-hole level of the valence band well. We have measured PL spectrum for different temperatures. In figure 4, the experimental and calculated integrated PL intensity are shown. We have observed an unexpected enhancement of the measured PL intensity with increasing temperature and a shift in the peak position when the applied bias is changed. All the theoretical curves in figure 4 were calculated with the same mobility obtained from the photocurrent and different values of the applied bias. The dashed line in the figure 4 are the calculations in the two transport limiting cases considering only one transport processes at a time: pure diffusion or pure drift. To calculate the characteristic lengths in each regime, the expression (6) was used. In both cases, the individual calculated value of $\lambda$ are 2 to 3 order of magnitude greater than when 
considering both mechanism acting together. Due to the high values of $\lambda$, the temperature dependence of the exponential term appearing in expression (10) is neglected compared with the temperature dependence of the quantum efficiency $\eta$. The theoretical model agree quit well with the experimental measurements and reproduce the shifting when the applied voltage is changed. This indicate the importance to take into account the effects of the photogenerated carriers in the contact and both transport mechanism, i.e. diffusion and drift.

\section{CONCLUSION}

We have studied the influence of minority carriers transport mechanism in the optical properties of double barrier diode. We have proposed a model to calculate the photocurrent and integrated photoluminescence intensity which take into account the diffusion, drift and tunneling process of photocreated holes in the contact. Within the approaches of our model we are able to reproduce qualitatively the temperature behavior of the experimental photocurrent and photoluminescence measurements. In particular, the anomalous enhancement observed in $G a A s-A l_{0.35} G a_{0.65} A s$ double barrier diodes with the increase of temperature was predicted in our model. Furthermore, we are able to simulate the minority holes mobility from the photocurrent measurements suggesting the use of the experimental technique as a probe of the carrier mobility.

\section{Acknowledgments}

FAPESP and PIBIC/CNPq/UFSCar for financial support.
[1] L. Pavesi, M. Guzzi, J. Appl. Phys. 75 4779-4842 (1994).

[2] J.F. Young, B.M. Wood, G.C. Aers, R.L.S. Devine, H.C. Liu, D. Landheer, M. Buchanan, A.J. SpringThorpe, and P. Mandeville, Phys. Rev. Lett. 60, 2085 (1988).

[3] N. Vodjdani, F. Chevoir, D. Thomas, D. Cote, P. Bois, E. Costard, and S. Delaitre, Appl. Phys. Lett. 55, 1528 (1989).

[4] K. Matsuda, T. Saiki, S. Nomura, M. Mihara, Y. Aoyagi, S. Nair, and T. Takagahara, Phys. Rev. Lett. 91, 177401 (2003).

[5] G.D. Gilliland, Mater. Sci. Eng. R-Rep. 18, 99 (1997).

[6] M.S. Skolnick, P.E. Simmonds, D.G. Hayes, A.W. Higgs, G.W.
Smith, A.D. Pitt, C.R. Whitehouse, H. Hutchinson, C.R.H White, L. Eaves, M. Henini, and O.H. Hughes, Phys. Rev. B 42, 3069 (1990).

[7] D.K. Ferry, Semiconductors, 1st Edition, Macmillan Publishing Company, New York, 1991.

[8] J.S. Blakemore, J. Appl. Phys. 53, R123 (1982).

[9] M.L. Lovejoy, M.R. Melloch, and M.S. Ludstrom, Appl. Phys. Lett. 67, 1101 (1995). 